\title{
Herramienta de robótica educativa basada en Lego Mindstorms y VEX Robotics mediante software 3D y diseño mecatrónico
}

Luis Carlos Correa Ortiz¹, Margarita María Vallejo Jiménez², John Jairo Martínez Puerta $^{3}$, Juan Alejandro Tru jillo Posada ${ }^{1}$

lcco@umanizales.edu.co, mvallejoj@sena.edu.co,jjmartinezp@sena.edu.co, juanalejandro@ umanizales.edu.co

${ }^{1}$ Facultad de Ciencias e Ingeniería, Universidad de Manizales, Carrera 9 No. 19-03, 17001, Manizales, Colombia.

${ }^{2}$ Sena Tecnoacademia Risaralda, Calle 73 Bis Carrera 21, 661001, Dosquebradas, Colombia

${ }^{3}$ Centro Atención Sector Agropecuario, Sena Risaralda. Sistema de Investigación Sennova, Carrera 8 \#26-79, 600002, Pereira, Colombia

DOI: 10.17013/risti.34.1-19

Resumen: La robótica propicia la adquisición de competencias técnicas en áreas tales como electrónica, informática, mecánica, y transversales como trabajo en equipo, resolución de problemas y pensamiento crítico. La mayoría de los currículos en las instituciones de educación básica, media y superior del país que utilizan robots educativos no tienen en cuenta el contexto ni el usuario final del robot, incluidas las dos instituciones de este estudio: Tecnoacademia Sena Risaralda y Universidad de Manizales. En este trabajo se expone el diseño, implementación y validación de una herramienta de software $3 \mathrm{D}$, que permitió configurar robots Lego Mindstorms EV3 y VEX Robotics EDR, para cumplir una tarea específica, teniendo en cuenta los requerimientos iniciales del usuario del robot. Se usaron herramientas de diseño mecatrónico basadas en el despliegue de la función de calidad QFD y Design Thinking. El software hará parte de los currículos de ambas instituciones en sus cursos de robótica.

Palabras-clave: Robótica educativa; Ingeniería; Sensores; Modelado 3D; Diseño mecatrónico.

\section{Educational robotics tool based on Lego Mindstorms and VEX Robotics using $3 D$ software and mechatronic design}

Abstract: Robotics encourages acquiring many technical skills in areas such as electronics, computer science, mechanics and also soft skills as teamwork, problem solving and critical thinking. Most of the curricula of elementary, middle and higher education institutions in Colombia that incorporate educational robots do not consider the context or the end user of the robot, including the two institutions involved in this study: Tecnoacademia Sena Risaralda and University of Manizales. In this work, design, implementation and testing of a $3 \mathrm{D}$ software tool is described. 
This tool allows configuring Lego Mindstorm EV3 and VEX Robotics EDR robots to compel a specific task, considering the initial requirements of the end user. Mechatronics design tools as QFD and design thinking was used. Finally, the developed software will be incorporated in robotic courses in both institutions.

Keywords: Educational Robotics; Engineering; Sensors; 3D Modeling; Mechatronic Design.

\section{Introducción}

La alfabetización científica es un proceso central en el desarrollo de las sociedades, existiendo razones tanto económicas como de movilidad social que invitan a fomentar y potenciar su desarrollo. Según la OCDE (Organización para la Cooperación y el Desarrollo Económicos), el alfabetismo científico no sólo involucra el tener conocimientos científicos sino la capacidad de aplicarlos funcionalmente en el medio donde se desenvuelven las personas (García \& Reyes, 2012). La educación STEM (Science, Technology, Engineering and Mathematics) creció de la preocupación por el bajo número de futuros profesionales en comparación con la alta demanda de profesionales STEM, en detrimento de la competitividad económica y educativa (Mizell \& Brown, 2017). La robótica educativa hace parte de la educación STEM (Silva-Ortigoza et al., 2014) y más recientemente STEAM (incorporando la palabra Arts) (OEA, 2016), el concepto se hace más amplio incluyendo innovación, creatividad y solución de problemas de la vida real (Ghitis \& Alba, 2014).

El resultado de una investigación en robótica educativa en diferentes partes del mundo arroja la tendencia de dos enfoques; el enfoque pedagógico, y el enfoque técnico. En el pedagógico se habla de la utilización de la robótica como una estrategia para la enseñanza de varias áreas escolares como matemáticas, cálculo, ciencias, informática, diseño, etc., y por otro lado en el enfoque técnico se centra en la llamada robótica multiagente o cooperativa y en la investigación para generar innovación en el hardware y software de robótica (Salas-Lòpez et al., 2012).

En el mundo se promueve el uso de la robótica como herramienta educativa en instituciones de educación básica primaria y secundaria, con el fin de fortalecer las competencias y el desempeño académico del educando mediante herramientas innovadoras que permitan una construcción más dinámica del conocimiento (CabelloOchoa \& Carrera-Farran, 2017) y además mediante STEM (Rivadeneira, 2019). Numerosas investigaciones demuestran el interés global por la inserción de herramientas robóticas en las aulas de clase. En 1975, en Francia, aparece una primera utilización con fines educativos de la robótica. En 1989, en México y 1998 en Costa Rica (Acuña, 2004). En España (Pinto, Barrera, \& Pérez, 2010), Chile (Monsalves, 2019) y Panamá (Moreno et al., 2012) se generan entornos de aprendizaje basados fundamentalmente en la acción de los estudiantes, quienes simulan fenómenos y mecanismos, diseñando y construyendo prototipos robóticos.

\subsection{Robótica educativa en Colombia}

En Colombia la Universidad Pedagógica Nacional lideró en 2004 proyectos para la formación de docentes en robótica educativa. En 2008, la Universidad del Cauca con 
"Plataforma de Robótica y Automática Educativa de Computadores para Educar". En Cali, el Colegio Bolívar, el Instituto Nuestra Señora de la Asunción INSA y la Fundación Gabriel Piedrahita Uribe han implementado talleres y en la Universidad Nacional de Colombia se realizó la construcción de un robot móvil didáctico para trabajo con niños de básica primaria (Pinto et al., 2010).

En la Universidad de Cundinamarca, con estudiantes de algunos colegios de Chía, se han construido "robots autónomos seguidores de línea" (Silva-Ortigoza et al., 2014) y se han conformado semilleros para desarrollar diferentes proyectos en áreas interdisciplinarias (Sánchez, 2003), creando la comunidad virtual "Mundo Robótica”, donde la comunidad educativa y académica interesada en la temática participa en un espacio virtual en el que pueden compartir sus experiencias, conocer nuevas prácticas de otros miembros de la red y construir conocimientos en torno a la robótica educativa (Bravo \& Forero, 2012).

Los primeros intentos por incorporar didácticas diferentes en el Sena (Servicio Nacional de Aprendizaje) se hicieron bajo la denominación de Tecnología Básica Transversal (TBT), desde finales de la década de los noventa, bajo la modalidad de formación por proyectos. La estrategia ha sido estructurar el aprendizaje de los alumnos a través de pequeños proyectos, a fin de impulsar el desarrollo de competencias transversales, soportadas en metodologías activas como pueden ser la resolución de problemas, la elaboración de proyectos, la simulación, el análisis o el estudio de casos entre las más empleadas (Sáez-Carreras, Bas-Peña, \& Bedmar-Moreno, 2007). Esta estrategia multidisciplinaria basada en proyectos tiene sus orígenes en la Universidad Aalborg en Dinamarca en 1974, en colaboración con la industria de ese entonces (Dym, Agogino, Eris, Frey, \& Leifer, 2005).

La formación correspondiente tanto a la educación secundaria como a los primeros semestres de la educación superior, tiene un enfoque diferente, no centrado en el perfil ocupacional de los estudiantes sino en el desarrollo de competencias en ciencia, tecnología e innovación, aprendizaje esencial hoy en día a tempranas etapas del desarrollo humano. Una de las metas más importantes de la educación colombiana es reforzar y ayudar en la incorporación de nuevas tendencias en el sector educativo y para tal fin se incorporan nuevas tecnologías en las instituciones educativas desde dos ámbitos: las TIC (Tecnologías de la Información y la Comunicación) y desde la tecnología como medio de construcción (Corchuelo, 2015).

El Ministerio de Educación Nacional de Colombia (2008) realizó una investigación dirigida a estudiantes de secundaria, proponiendo un modelo de ambientes de aprendizaje en robótica, concluyendo que no existe un consenso a nivel mundial sobre características estándar. Afirma además que formar en tecnología es mucho más que ofrecer una capacitación para manejar artefactos y expone algunos desafíos:

- Motivar mediante curiosidad científica y tecnológica, mostrando pertinencia en la realidad local y su contribución a la satisfacción de necesidades.

- Reconocer el saber tecnológico como solución a problemas que contribuyen a la transformación del entorno.

- Participación de los individuos en la evaluación y control de la tecnología en la sociedad, desarrollando una reflexión crítica. 
- Reconocer la existencia de varios enfoques para la solución de problemas de tecnología, como el diseño, la innovación, la detección de fallas y la investigación.

- Brindar a las personas herramientas para participar asertivamente en su entorno.

Existen currículos que enseñan a diseñar, construir y programar kits de robótica, como resultado de la experimentación de la robótica pedagógica en el mundo. En Colombia y especialmente en la zona cafetera algunos currículos están basados en kits robóticos pertenecientes a la Tecnoacademia Sena Risaralda (Vallejo-Jiménez, 2018) y a la Universidad de Manizales, tales como Lego Mindstorm EV3®) (Chetty, 2015) y VEX Roboticsß. Estos currículos, basados en la metodología STEM (Correll, Wailes, \& Slaby, 2014), muchas veces se limitan a la enseñanza del funcionamiento del robot, pero sin enfocarse en dar solución a las problemáticas o necesidades del entorno, mediante el desarrollo de prototipos iniciales para ello, excluyendo a las competencias, que se dan cuando los estudiantes poseen dominio sobre las plataformas robóticas.

En este ambiente de aprendizaje innovador los estudiantes ocupan la mayor parte del tiempo simulando fenómenos y mecanismos, diseñando y construyendo prototipos que son representaciones micro de la realidad tecnológica circundante, o son sus propias invenciones (Vallejo-Jiménez, Martínez-Puerta, Bedoya-Agudelo, \& Salgado, 2018). La puesta en marcha de un proyecto de robótica requiere del conocimiento de diversas áreas, pues el robot es un artefacto mecatrónico que surge de la combinación de mecánica, electrónica y ciencias de la computación, campos que se influencian mutuamente y que finalmente son dotados con cierta inteligencia o funciones autónomas (Isermann, 2008). Todas las áreas influyen en las demás, esto significa que se debe llevar a cabo ingeniería simultánea con el objetivo de diseñar un sistema global integrado y también crear efectos sinérgicos (Isermann, 2008), (Odorico, 2005).

\subsection{Metodología para construcción de prototipos}

Cuando se habla de diseñar soluciones a través de prototipos robóticos, pueden resultar productos ineficientes, incremento de costos por ajustes y largos períodos de desarrollo del producto, todo esto cuando el diseño no se realiza de manera concurrente (Steimer, Fischer, \& Aurich, 2017). Es allí donde se aplican las metodologías de diseño de productos, en especial de diseño mecatrónico, que permiten estimar tiempos de diseño y prototipado rápido debido a las exigencias de la industria y al ciclo de vida cada vez más corto de los productos (De Silva \& Behbahani, 2013).

Existen diversas metodologías y herramientas para el diseño industrial de prototipos (Dym et al., 2005) (Brown, 2008) (Sena, 2015) (Gómez-González, Lecuona-López, \& Hernández-Monsalve, 2016). También existen metodologías centradas en el diseño mecatrónico (Dode, Greig, Zolfaghari, \& Neumann, 2016) (Corchuelo, 2015), entre ellas la ingeniería simultánea o concurrente (Macas, 2017); fábrica digital (Kuhn, 2006); metodología de diseño para sistemas mecatrónicos, guía VDI 2206 (Dode et al., 2016) (Gausemeier, Dorociak, Pook, Nyßen, \& Terfloth, 2010); modelo de tres ciclos de ingeniería de producto (Kurdve, Zackrisson, Wiktorsson, \& Harlin, 2014); desarrollo de doble hélice de sistemas de producción y productos (Pessôa \& Trabasso, 2016); ingeniería de sistemas basada en modelos (Steimer et al., 2017); Cociente de Diseño Mecatrónico, 
MDQ, por sus siglas en inglés (De Silva \& Behbahani, 2013); métodos ágiles (Cooke, 2012) (Edin Grimheden, 2013); despliegue de la función de calidad y Casa de Conceptos HoC (por sus siglas en inglés) (Punz, Hehenberger, Follmer, \& Zeman, 2011) (Hassan, Siadat, Dantan, \& Martin, 2010) y Design Thinking (Brown, 2008).

No existe un consenso que defina cual metodología es mejor, pues estas se ajustan según el objetivo o prototipo que se desee construir, pero la mayoría conciben ciertos pasos o etapas en común para la construcción de un artefacto mecatrónico (Carvajal, 2013):

- Diseño conceptual: Se define la función de cada disciplina por módulos de diseño mecánico, eléctrico-electrónico y diseño de software; en cada una se definen parámetros y variables de diseño.

- Modelado: Se realiza el modelado gráfico y/o modelado matemático a través de software especializado para cada disciplina.

- Simulación: Se construye la simulación en un ambiente virtual, por módulos disciplinares o integrados parcialmente, dando como resultado la simulación del prototipo mecatrónico a través de software especializado.

- Integración: Se define la integración de los módulos disciplinares, optimización del diseño integrado, aplicación de software especializado, desarrollo del diseño en ambiente de Ingeniería concurrente con ajustes y pruebas de la función transdisciplinar del producto.

En las metodologías verificadas se observa poca uniformidad para la elección de las herramientas que permitan llevar a cabo las etapas anteriores para la construcción de prototipos, existiendo una gran variedad de ellas.

Uno de los primeros intentos en el país para desarrollar y adaptar herramientas concretas para el desarrollo de prototipos lo hace el Sena en su programa Tecnoparque Colombia, quienes apropiaron desde el año 2015 una variante del enfoque Design Thinking (Dym et al., 2005)(Sena, 2015) mediante el cual se desarrollan prototipos con base en una necesidad existente y bajo la premisa que el diseño en ingeniería puede verse materializado mediante la aplicación de herramientas definidas de Diseño Industrial. En otras instituciones como la Universidad de Manizales, estos procesos de construcción de artefactos mecatrónicos están regidos por el currículo existente y no por una metodología estándar, dando como resultado que los estudiantes construyan exitosamente estructuras robóticas y programan sus artefactos de manera correcta, pero sin un propósito, norte o contexto definido, para luego de ser construido, encontrarle una utilidad.

En las diferentes sedes de las Tecnoacademia Sena presentes en el país se vienen desarrollando procesos similares en la concepción de un robot para solucionar una necesidad usando algunas herramientas Design Thinking, pero no se tienen establecidas unas herramientas estándar en todas las sedes que permitan que el diseño mecatrónico sea óptimo. Además, las limitaciones de presupuesto hacen que la integración con el hardware de los robots esté al alcance de pocas instituciones educativa de educación básica, media e incluso superior. Una de las posibilidades es centrarse en la realización de una herramienta de software centrada en la etapa de Integración descrita anteriormente, en donde no sea necesario tener el hardware del robot al alcance de los estudiantes para desarrollar la solución. 
El objetivo del estudio es implementar una herramienta de software $3 \mathrm{D}$ que permita configurar robots Lego Mindstorms EV3 y VEX Robotics EDR para cumplir una tarea específica teniendo en cuenta los requerimientos iniciales del usuario del robot. Para cumplir este objetivo se hace necesario como primera medida levantar los requerimientos tanto en términos de la herramienta robótica, como del software a desarrollar, para posteriormente implementar la herramienta y finalmente validarla en el marco de los cursos de robótica de ambas instituciones.

La herramienta plantea una necesidad en un contexto determinado y a partir de esta y de los requerimientos que el cliente tenga (quien usará el robot) se construye la solución, al contrario de lo que se hacia anteriormente, buscar el sentido al artefacto robótico ya construido. Puede usarse adicionalmente tanto como instrumento de enseñanza aprendizaje en un curso de robótica en el cual no exista el hardware de robots Lego y VEX como una herramienta de apoyo centrada en las necesidades del usuario. El software, además de recoger los requerimientos del usuario y transformarlos en criterios técnicos, posee un tutorial de las partes del robot y su funcionamiento. Es allí donde se convierte en el primer acercamiento al funcionamiento de los robots, como sensibilización e introducción a los cursos de robótica de ambas instituciones. El aprendizaje significativo en los educandos tiene que ver con el acercamiento a metodologías de diseño mecatrónico centrado en el usuario y en las problemáticas, usando herramientas como QFD. No se pretende encasillar el software para que sea usado en los cursos de robótica de una manera particular, se desean exponer sus ventajas para que pueda ser aprovechado de la mejor forma posible en los cursos.

\section{Materiales y Métodos}

La herramienta $3 \mathrm{D}$ se diseñó en la plataforma de desarrollo en tiempo real Unity® (https://unity.com/es) como un juego para el sistema operativo Windows, en donde inicialmente al jugador o usuario del robot se le plantea la necesidad específica de encontrar un objeto mediante Lego Mindstorms EV3 o VEX EDR. En primer lugar, el software le hace once preguntas al usuario relacionadas con lo que desea que haga su robot para resolver el reto: ¿Es importante que su robot se mueva en todas direcciones? ¿O que el robot evada obstáculos? ¿Piensa que su robot debe buscar el objeto en un escenario cerrado o interior? ¿O desea que sea en uno exterior?, a las cuales el usuario debe responder asignando valores en una escala de cero a cinco a cada pregunta. Un valor de cero significa que no es importante que el robot posea esa característica y uno de cinco significa que la considera indispensable.

A continuación, se le plantea la elección del robot en el escenario que se planteó anteriormente (interior o exterior). Puede elegir entre una configuración de Lego Mindstorms EV3 o de VEX EDR. Cada uno de los robots fueron diseñados pieza por pieza, por lo tanto, antes de la elección el jugador puede analizarlo, podrá manipularlo mediante vistas, además de ver el nombre de las partes que lo componen y de ver una corta descripción de lo que hace cada una.

Luego de elegir el robot, se presenta una animación del comportamiento del robot en el escenario correspondiente. La finalidad de la herramienta no es programar los movimientos del robot para que puedan visualizarse, es la elección de los sensores y 
actuadores adecuados para el robot, dependiendo de los deseos del jugador. El proyecto se realizó en Unity pues permitió integrar las piezas del diseño 3D de otro tipo de software.

\subsection{Etapas}

El software fue concebido para cursos introductorios de robótica basada en Lego y VEX, usando herramientas de diseño mecatrónico, centradas en la problemática. Los estudiantes de la Tecnoacademia Sena Risaralda pertenecen a los grados $7^{\circ}$ a $10^{\circ}$ de educación secundaria de los colegios de la región. En este caso la formación es básica y no involucra conceptos de cinemática, dinámica o incluso electrónica avanzada, siendo las 140 horas de formación por año insuficientes para este desarrollo. Por parte de la Universidad de Manizales, los estudiantes pertenecen a los tres primeros semestres de Ingeniería de Sistemas y Telecomunicaciones y el curso contempla 64 horas. En ambos casos, la metodología de trabajo se basa en el desarrollo de competencias en ciencia, tecnología e innovación con las metodologías STEAM y Design Thinking, adaptadas e implementadas por ambas instituciones.

El proyecto se realizó en dos etapas, cada una de ellas con una metodología diferente:

- $\quad$ Etapa 1: Se establecieron los requerimientos o deseos del usuario de los robots y estos se tradujeron en criterios técnicos para su construcción, todo a través de la metodología de diseño mecatrónico basada en Despliegue de la Función de Calidad o QFD.

- Etapa 2: Estos criterios técnicos fueron el insumo para la realización del software $3 \mathrm{D}$ en Unity a través de metodología Design Thinking de la Red Tecnoparque Sena.

A continuación, se enumeran las diferentes actividades que se llevan a cabo en cada etapa.

\subsection{Etapa 1}

Para las plataformas de robótica educativa (Lego Mindstorms EV3 y VEX EDR) disponibles tanto en la Tecnoacademia Sena Risaralda como en la Universidad de Manizales, se establecieron dos configuraciones, que corresponden a su vez a dos escenarios (interiores y exteriores), según la Tabla 1.

\begin{tabular}{lccl}
\hline $\begin{array}{l}\text { Plataforma } \\
\text { Robótica }\end{array}$ & Configuración de Robot & Escenario & $\begin{array}{l}\text { Características } \\
\text { Escenario }\end{array}$ \\
\hline $\begin{array}{l}\text { Lego Mindstorms } \\
\text { EV3 }\end{array}$ & Lego1 & Interiores & $\begin{array}{l}\text { Espacio conocido y cerrado } \\
\text { como casas, talleres, } \\
\text { almacenes, bodegas, etc. }\end{array}$ \\
\hline VEX EDR & Vex1 & Exteriores & $\begin{array}{l}\text { Espacio desconocido, } \\
\text { abierto y abrupto como el } \\
\text { campo, canchas, estadios, } \\
\text { etc. }\end{array}$ \\
\hline $\begin{array}{l}\text { Lego Mindstorms } \\
\text { EV3 }\end{array}$ & Lego1 & Vex1 & \\
\hline VEX EDR & & & \\
\hline
\end{tabular}

Tabla 1 - Plataforma Robótica - Escenario. 
La finalidad de la Etapa 1 fue establecer las características técnicas para el robot en cada escenario, a partir de los requerimientos o deseos de los clientes, que correspondieron a las preguntas iniciales del juego, respondidas por los estudiantes. Estas respuestas fueron el insumo de la matriz de la metodología de diseño mecatrónico QFD, cuyos resultados arrojados correspondieron a los requerimientos técnicos de los robots (sensores, actuadores, etc.).

Para ello se hizo un diseño de experimento, de la siguiente manera:

1. Objeto del experimento: Obtener los criterios técnicos del robot para cada uno de los escenarios (interiores y exteriores).

2. Fuentes de variación: Los pesos asignados a los requerimientos de los clientes, valores de o (sin importancia) a 5 (importante).

3. Factores de tratamiento: factores cualitativos reflejados en la tabla 2.

\begin{tabular}{|c|c|c|c|}
\hline $\begin{array}{l}\text { Categoría de los } \\
\text { factores }\end{array}$ & \# & Factores de tratamiento & Niveles \\
\hline $\begin{array}{l}\text { Facilidad de } \\
\text { manipulación por } \\
\text { parte del usuario }\end{array}$ & 1 & $\begin{array}{l}\text { Manipulación del robot por } \\
\text { parte del usuario }\end{array}$ & $\begin{array}{l}\text { Fácil (se puede tomar con ambas } \\
\text { manos) y Difícil (no se puede } \\
\text { manipular con ambas manos) }\end{array}$ \\
\hline \multirow{3}{*}{$\begin{array}{l}\text { Facilidad de } \\
\text { programación }\end{array}$} & 2 & Tipo de interfaz & Amigable o poco amigable \\
\hline & 3 & $\begin{array}{l}\text { Cumplimiento de tarea con } \\
\text { orden inicial }\end{array}$ & Cumple o no cumple \\
\hline & 4 & $\begin{array}{l}\text { Posibilidad de elección del } \\
\text { modo de la tarea }\end{array}$ & Cumple o no cumple \\
\hline \multirow{5}{*}{$\begin{array}{l}\text { Facilidad de } \\
\text { movimiento }\end{array}$} & 5 & $\begin{array}{l}\text { Movimiento en cualquier } \\
\text { dirección }\end{array}$ & Se mueve o no se mueve \\
\hline & 6 & Evasión de obstáculos & Evade o no evade \\
\hline & 7 & $\begin{array}{l}\text { Movimiento autónomo por } \\
\text { un tiempo prudente }\end{array}$ & Se mueve o no se mueve \\
\hline & 8 & Movimiento en exteriores & $\begin{array}{l}\text { Se elige el escenario exterior o no } \\
\text { se elige }\end{array}$ \\
\hline & 9 & Movimiento en interiores & $\begin{array}{l}\text { Se elige el escenario interior o no } \\
\text { se elige }\end{array}$ \\
\hline \multirow{2}{*}{$\begin{array}{l}\text { Interacción con el } \\
\text { ambiente }\end{array}$} & 10 & Reacción a la luz & Reacciona o no reacciona \\
\hline & 11 & Reacción a colores & Reacciona o no reacciona \\
\hline
\end{tabular}

Tabla 2 - Diseño de Experimento.

4. Factores externos:

- Marca del robot: Lego o VEX. No se puede medir al inicio.

- Grado de escolaridad de la unidad experimental: Colegio o cursando universidad, si se puede establecer al inicio.

5. Unidades experimentales:

- Cursos 1.1, 1.2, 1.3 y 1.4 de Estudiantes de Robótica de la Tecnoacademia Sena Risaralda

- Curso 2.1 de estudiantes de Robótica de la Universidad de Manizales.

- Bloques: 5 (cada uno de los cursos).

6. Regla de asignación: Por bloque 
7. Medidas que se realizarán: Variables respuesta: 12 criterios técnicos para la configuración en interiores y 12 criterios técnicos para la configuración en exteriores, medidos en porcentaje de importancia o peso, reflejados en la tabla 3.

\begin{tabular}{lc}
\hline 1 & Movimiento por ruedas \\
\hline 2 & Movimiento por orugas \\
\hline 3 & Baterías recargables \\
\hline 4 & Sensor de color \\
\hline 6 & Sensor ultrasónico \\
\hline 7 & Sensor push-button (final de carrera) \\
\hline 8 & Girosensor \\
\hline 9 & Monitoreo por computadora en tiempo real \\
\hline 10 & Programación mediante software por computador \\
\hline 11 & Sensor de luz \\
\hline
\end{tabular}

Tabla 3 - Variables Respuesta.

8. Instrumento de medida: Matriz de Despliegue de la Función de Calidad QFD

9. Experimento piloto: Tecnoacademia Sena Risaralda: Cuatro cursos con cuatro subgrupos cada uno. Universidad de Manizales: Un curso con cuatro subgrupos.

10. Modelo matemático: Relaciones, pesos y fórmulas propios de la QFD (Hassan et al., 2010).

11. Análisis estadístico: Media de las variables respuesta por cada escenario. Se eligieron los 6 criterios con mayor ponderación relativa, por escenario, para la construcción de los modelos Lego1, Lego2, Vex1 y Vex2.

\subsection{Etapa 2}

La metodología que se toma es la establecida por la Red Tecnoparque del SENA (Sena, 2015) y adaptada de IDEO (Brown, 2008) para el desarrollo de prototipos:

1. Inspiración: Se establece el escenario PON: problema, oportunidad y/o necesidad, el cual corresponde a la realización del software con robots. Se realiza una vigilancia tecnológica en tres temas prioritarios: software para la programación de la herramienta, piezas o robots modelados, software para modelar las piezas faltantes.

2. Ideación: Se desarrolla el concepto integral de diseño de software, se bosqueja la parte visual y los menús, además se establecieron los requerimientos de interacción con el usuario y las posibles salidas con sus métricas, además de las configuraciones de los robots.

3. Prototipado: Se realiza la programación, se modelan las partes de los robots faltantes y se realizan las animaciones finales. Luego se hace tanto la prueba del software como la validación del prototipo con los usuarios. En todas las etapas se proponen cambios, además de compartir las historias y experiencias aprendidas en el proceso de desarrollo de la solución. 


\section{Resultados}

\subsection{Etapa 1}

Inicialmente se realizó un inventario de las características de los robots Lego Mindstorms EV3 y del VEX EDR, en cuanto a sensores y actuadores, los cuales fueron recopilados por los estudiantes de ambas instituciones al inicio del curso.

A medida que los estudiantes adquirieron las competencias necesarias en el manejo de las plataformas robóticas se les presentaron ambos escenarios y se les preguntó cuáles eran las características relevantes que un robot debería tener para cumplir una misión cualquiera en cada escenario.

Luego, con ayuda de los docentes, se agruparon estas características en categorías y se eliminaron las que eran similares o repetidas, quedando las categorías descritas en la tabla 2.

Alinicio del curso los estudiantes realizaron un inventario delos elementos que conforman a las plataformas LEGO EV3 y VEX; posteriormente identificaron las características y elementos comunes a ambas plataformas y se eligieron las más relevantes. Estas etapas se describen en la figura 1.

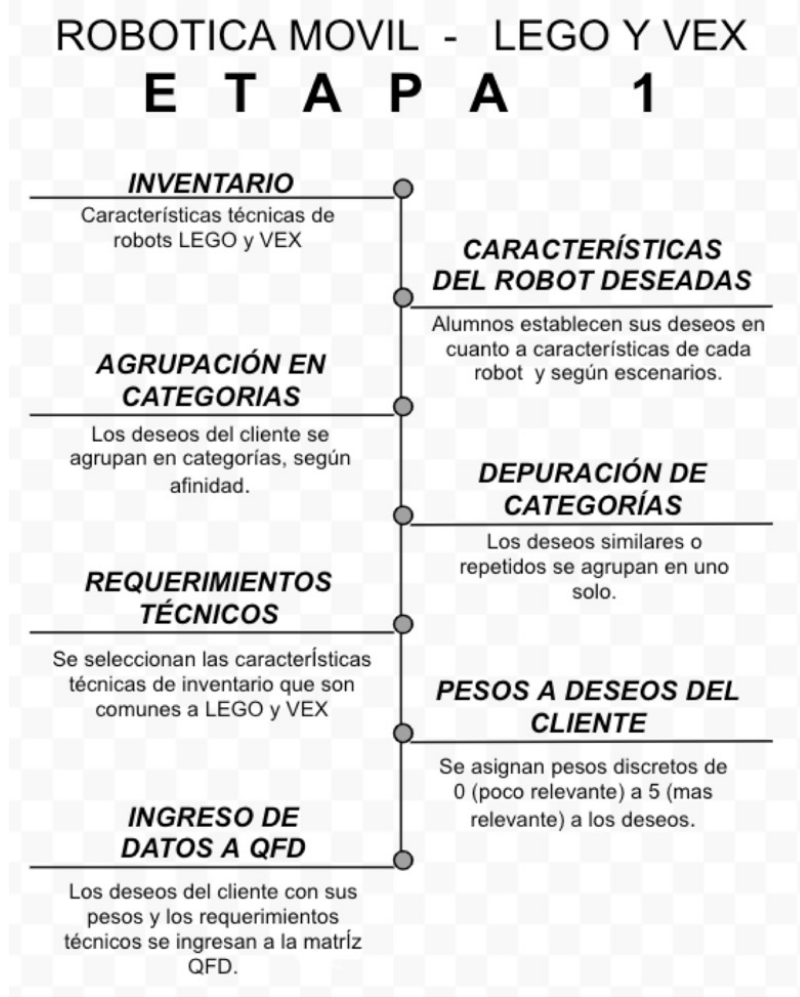

Figura 1 - Etapas contempladas en el proyecto. 
A cada bloque asignado en el diseño de experimentos se le pidió que asignaran grados de importancia o pesos a los deseos del cliente, por tipos de escenarios (interiores y exteriores), tomando como valores o a 5. Luego se realizó el promedio de estos valores, dando como resultado dos grupos de deseos del cliente priorizados, uno para robots que se mueven en interiores y otro para robots que se mueven en exteriores (tabla 4).

\begin{tabular}{|c|c|c|c|}
\hline No. & Requerimientos del Cliente & Interiores & Exteriores \\
\hline 1 & Que sea fácil de manipular & 5 & 4 \\
\hline 2 & Que tenga una interfaz amigable para darle órdenes & 5 & 4 \\
\hline 3 & Que cumpla su tarea con una orden inicial & 3 & 5 \\
\hline 4 & Que pueda elegirse el modo de hacer la tarea (customización) & 3 & 2 \\
\hline 5 & Que se mueva en cualquier dirección & 4 & 3 \\
\hline 6 & Que evada obstáculos & 3 & 4 \\
\hline 7 & Que se mueva de manera autónoma por un tiempo prudente & 5 & 5 \\
\hline 8 & Que se mueva en exteriores & o & 5 \\
\hline 9 & Que se mueva en interiores & 5 & o \\
\hline 10 & Que reaccione con la luz & 1 & 4 \\
\hline 11 & Que responda a los colores & 4 & 1 \\
\hline
\end{tabular}

Tabla 4 - Pesos promedio de los requerimientos del cliente por cada tipo de escenario.

Esta información de pesos es el insumo inicial de la matriz de diseño mecatrónico QFD. Al final, a cada bloque se le presentaron dos posibles escenarios, uno de exteriores y otro de interiores, cada uno con un robot Lego Mindstorm EV3 y otro VEX que den respuesta a los requerimientos planteados desde el inicio.

Luego de ingresar los deseos del cliente, se ingresan los requerimientos técnicos en la matriz QFD. Posteriormente se obtienen las siguientes gráficas (figuras 2 y 3), con los pesos promedio de los requerimientos técnicos para cada configuración (tablas 5 y 6):

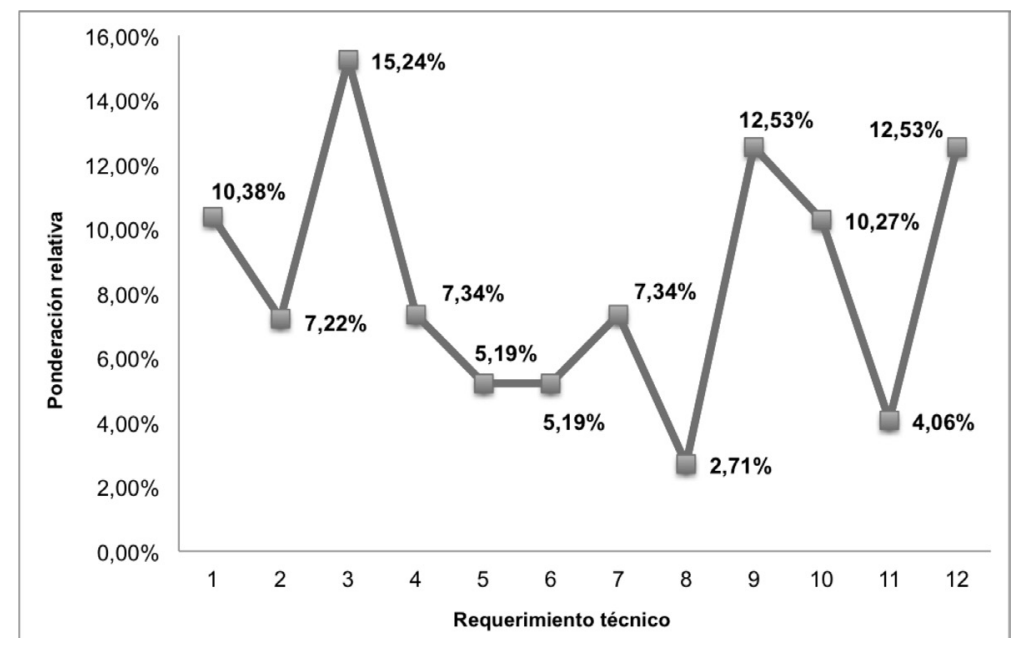

Figura 2 - Ponderación relativa (interiores). 


\begin{tabular}{ccc}
\hline No. & Ponderación relativa & Criterio Técnico \\
\hline 3 & $15,24 \%$ & Baterías recargables \\
\hline 9 & $12,53 \%$ & Monitoreo por computadora en tiempo real \\
\hline 12 & $12,53 \%$ & Programación in situ (en el mismo robot) \\
\hline 1 & $10,38 \%$ & Ruedas (conf. diferencial) \\
\hline 10 & $10,27 \%$ & Programación mediante software por computador \\
\hline 7 & $7,34 \%$ & Sensor de color \\
\hline 4 & $7,34 \%$ & Orugas (2 orugas) \\
\hline 2 & $7,22 \%$ & Sensor ultrasónico \\
\hline 5 & $5,19 \%$ & Sensor push- button \\
\hline 6 & $5,19 \%$ & Sensor de luz \\
\hline 11 & $4,06 \%$ & Actuador con motor mediano \\
\hline 8 & $2,71 \%$ & \\
\hline
\end{tabular}

Tabla 5 - Ponderación relativa (interiores).

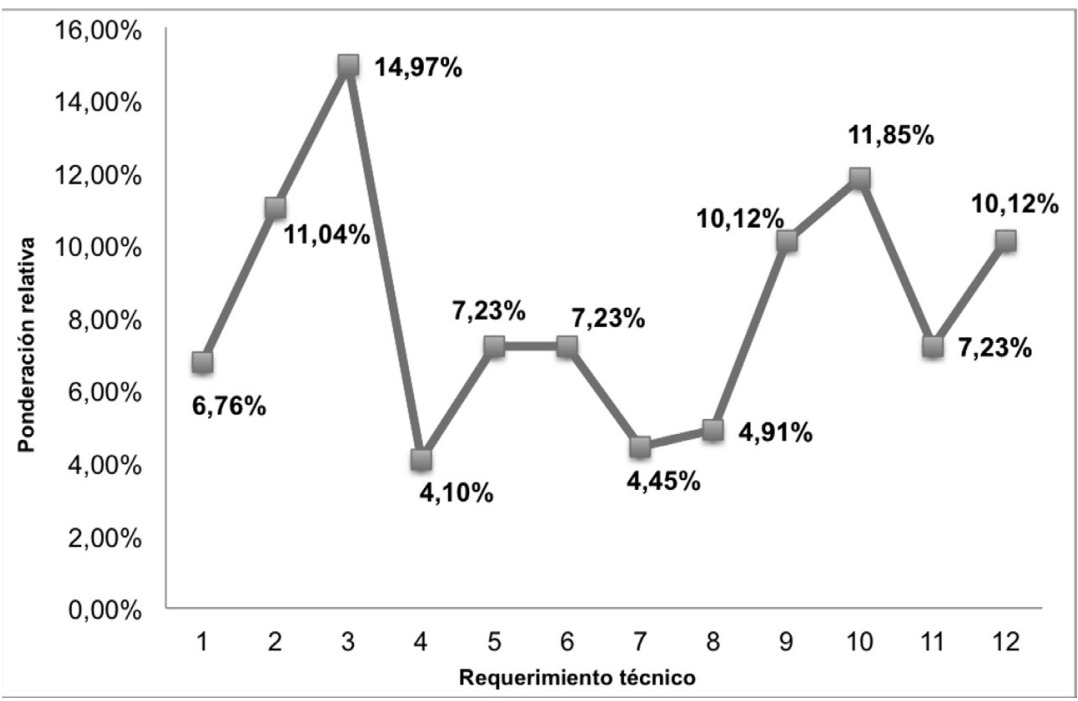

Figura 3 - Ponderación relativa (exteriores).

\begin{tabular}{ccc}
\hline No. & Ponderación relativa & Criterio Técnico \\
\hline 3 & $14,97 \%$ & Baterías recargables \\
\hline 10 & $11,85 \%$ & Programación mediante software por computador \\
\hline 2 & $11,04 \%$ & Orugas \\
\hline 9 & $10,12 \%$ & Monitoreo por computadora en tiempo real \\
\hline 12 & $10,12 \%$ & Programación in situ (en el mismo robot) \\
\hline
\end{tabular}




\begin{tabular}{ccc}
\hline No. & Ponderación relativa & Criterio Técnico \\
\hline 5 & $7,23 \%$ & Sensor ultrasónico \\
\hline 6 & $7,23 \%$ & Sensor push-button \\
\hline 11 & 7,23 & Sensor de luz \\
\hline 1 & $6,76 \%$ & Ruedas (conf. diferencial) \\
\hline 8 & $4,91 \%$ & Actuador con motor mediano \\
\hline 7 & $4,45 \%$ & Girosensor \\
\hline 4 & $4,10 \%$ & Sensor de color \\
\hline
\end{tabular}

Tabla 6 - Ponderación relativa (exteriores).

\subsection{Etapa 2}

1. Inspiración: Luego de modelar el problema y la vigilancia tecnológica, se elige Unity para realizar la herramienta y se encuentran pocas piezas modeladas para los robots. Se eligieron Solidworks $\AA$ e Inventor $\AA$ para modelar las piezas faltantes, cuyo resultado se observa en la Figura 4.

2. Ideación: Se realiza el bosquejo para los escenarios interiores y exteriores (Figura 5) y se establecen las preguntas que se le hacen al jugador, junto con las respuestas y sus métricas. Se elige para interiores las configuraciones de robots Lego "Base motriz" y VEX EDR "Clawbot” (Figura 6). Para exteriores se realiza la configuración "tanque" de Lego y para VEX EDR se usa "Clawbot” tomando como variante el desplazamiento por orugas.

3. Prototipado: Se realizó la programación y se modelaron las partes de los robots faltantes, al igual que las animaciones finales. Luego se hizo un testeo del software y luego el prototipo se validó funcionalmente con un grupo de estudiantes de ambas instituciones.

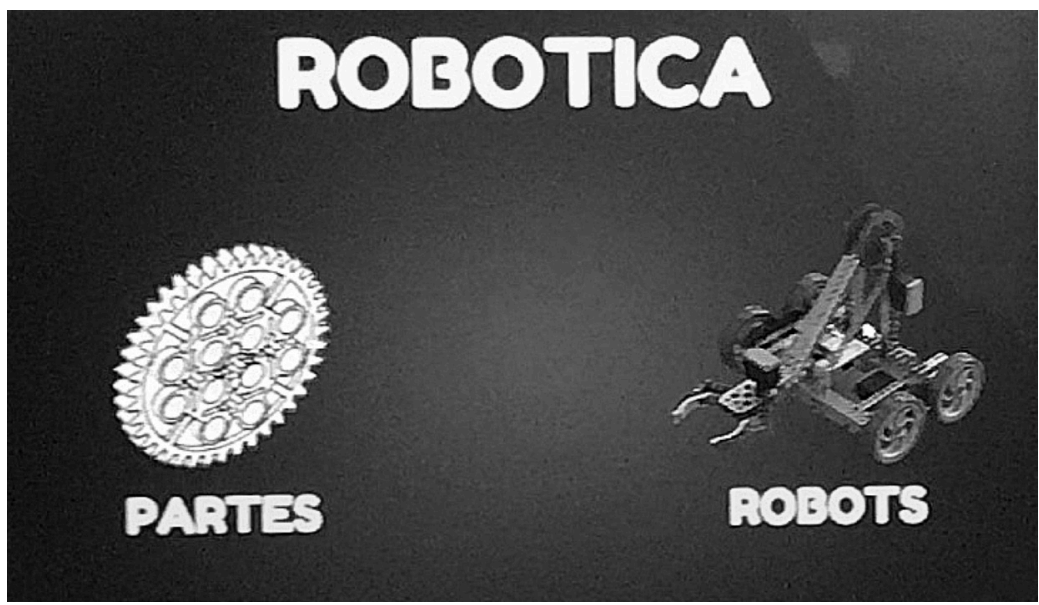

Figura 4 - Menú interactivo del Software. 


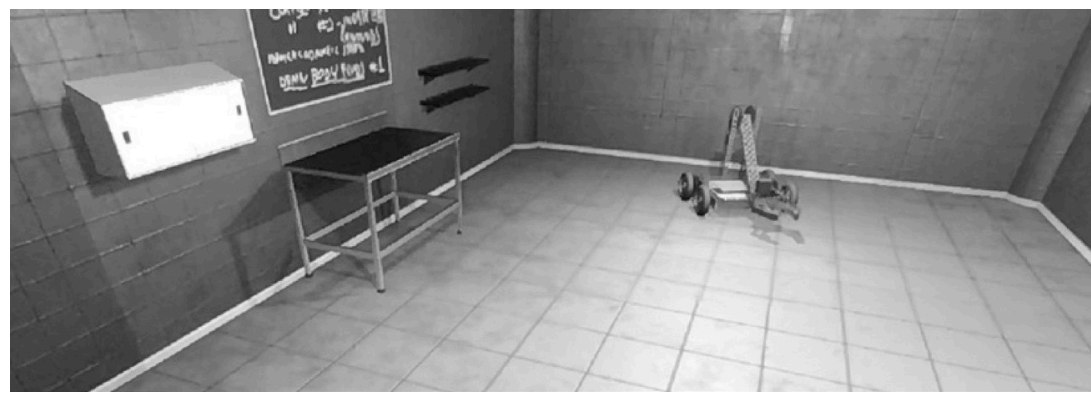

Figura 5 - Escenario de interiores para robot VEX.

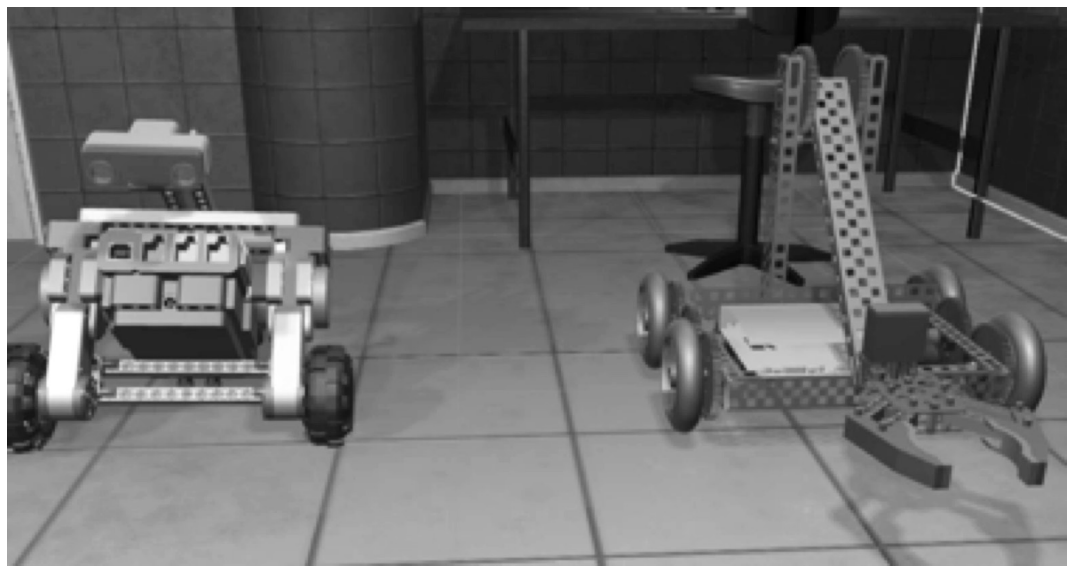

Figura 6 - Configuraciones de robots para interiores. Lego y VEX.

\section{Discusión}

Es importante aclarar que la metodología de despliegue de la función de calidad QFD es un proceso secuencial, el cual debe ser preparado y planeado mediante pesos y relaciones. Por ejemplo, luego de que se establecieron los requerimientos iniciales del cliente mediante las preguntas, se estableció el grado de relación que existe entre cada requerimiento del cliente versus cada requerimiento técnico, usando una escala ponderada no lineal para enfatizar claramente la importancia de los valores. Los grados de relación se clasificaron en relación fuerte, moderada y débil, con valores de 9, 3 y 1 respectivamente. Si no existía relación, simplemente el campo se dejó en blanco. Además, se estableció una relación fuerte, débil o nula entre cada par de características técnicas, es decir, de que manera influía una característica técnica en otra. Todo esto es propio de la metodología QFD y debe realizarse por expertos conocedores de las 
herramientas robóticas. En este caso, estos valores fueron elegidos por los docentes de las materias de robótica de ambas instituciones.

Se observa en los resultados del escenario interiores que la ponderación relativa más alta corresponde a las baterías recargables, por lo tanto, es un requisito esencial para tener en cuenta en la configuración de los robots Lego y VEX. Entre mas baterías de repuesto posea el robot, mas autonomía tendrá. También se aprecia que el movimiento debe ser por ruedas, más que por orugas y que los sensores principales que deben tener son el giroscopio y el sensor de color. El giroscopio puede ser útil en interiores donde el espacio es conocido y por lo tanto se podrían programar sus movimientos más precisamente. También está la opción de incorporar el sensor ultrasónico, en la medida en que el entorno en interiores no sea completamente conocido.

En el escenario de exteriores, la ponderación relativa más alta corresponde a las baterías recargables, por lo tanto, es un requisito esencial en la configuración de los robots Lego y VEX también para esta configuración. Se observa que el movimiento debe ser por orugas, más que por ruedas, lo cual es compatible con el movimiento en exteriores en terrenos difíciles o desconocidos. Además, los sensores principales relevantes son el sensor ultrasónico y el sensor push-button, teniendo en cuenta que el robot debe evadir obstáculos en un entorno desconocido. También está la opción de incorporar el sensor de luz, en la medida en que el entorno en exteriores tenga poca luz día.

Se tiene planeado repetir el experimento, esta vez teniendo en cuenta factores externos que se puedan medir en el inicio, como el tipo de robot o el grado de escolaridad de la unidad experimental, debido a que es diferente para los estudiantes de ambas instituciones, pues se quiso tener primero un referente, debido a que los grupos tenían el mismo grado de conocimiento respecto a los robots. Además, se estudia la posibilidad de incorporar elementos de realidad virtual en la herramienta. Sería interesante realizar el experimento con estudiantes que no hayan usado previamente las herramientas de robótica educativa y conocer las diferentes configuraciones que pueden realizar. De la misma manera, se contempla incluir en el desarrollo la posibilidad de dar cumplimiento a los requerimientos haciendo uso de plataformas robóticas adicionales de acuerdo con la disponibilidad de las mismas.

Es pertinente aclarar que, aunque se encontraron aplicaciones de software relacionadas con el funcionamiento y simulación de diferentes tipos de robot, no se encontraron herramientas de software de diseño mecatrónico para robótica móvil. Un referente es el programa V-rep (http://www.coppeliarobotics.com) el cual permite simulaciones de cada una de las piezas que forman un robot, como motores, orugas, hélices o patas, se puede controlar el movimiento del robot y configurar sus sensores como si se tratase de una máquina real, pero aprender su manejo requiere un tiempo considerable y no utiliza herramientas de diseño mecatrónico para la selección de los accesorios de cada robot. Una comparación de diferentes tipos de software de robots lo hace el grupo de Robótica Educativa de la Universidad de Alcalá (http://www.roboticaeducativa.org) en donde identifican diferentes características de algunos de los softwares mas populares en robótica, pero no se halló tampoco referencia a la incorporación de herramientas de diseño mecatrónico en estas aplicaciones. 
La identificación, aplicación y validación de diferentes herramientas de diseño mecatrónico mediante el uso de la robótica pedagógica para solucionar una necesidad, se convierte en el punto de partida de una educación que verdaderamente logre un impacto y un cambio de mentalidad, aprovechando los escenarios y la infraestructura existentes. Esto permitirá identificar las fortalezas, gustos e intereses de los educandos, adquiriendo las competencias necesarias para que puedan continuar con su formación en niveles superiores o realizar trabajos relacionados con la ciencia y la tecnología que le sean útiles a la comunidad.

El hecho de que los docentes de ambas instituciones estén utilizando técnicas de diseño centradas en lo humano para entender mejor a sus estudiantes, hace que los participantes se sientan comprometidos a cambiar su ambiente de aprendizaje. Además, en la medida que el docente se convierta en el diseñador de su propia experiencia en el aula, profesionaliza su rol y permite un cambio valioso y autentico, impulsado por las necesidades de los estudiantes, en vez de hacerlo por un mandato administrativo.

\section{Referencias}

Acuña, A. (2004). Robótica y aprendizaje por Diseño. Revista La Educ@ción de la OEA (Organización de los Estados Americanos), 139, 1-12. http://www.educoas.org/ portal/bdigital/lae-ducacion/139/pdfs/139pdf7.pdf

Bravo, F., \& Forero, A. (2012). La robótica como un recurso para facilitar el aprendizaje y desarrollo de competencias generales. Teoría de la Educación: Educación y Cultura en la Sociedad de la Información, 13(2), 120-136. Recuperado de: http:// www.redalyc.org/articulo.oa?id=201024390007

Brown, T. (2008). Design Thinking. Harvard Business Review, 86(9), 62-72.

Cabello-Ochoa, S., \& Carrera-Farran, F. X. (2017). Diseño y validación de un cuestionario para conocer las actitudes y creencias del profesorado de educación infantil y primaria sobre la introducción de la robótica educativa en el aula. Electrónica de Tecnología Educativa, 60, 1-22. doi: 10.21556/edutec.2017.60.871

Carvajal, J. (2013). Revisión y análisis de diseño mecatrónico para diseño curricular transdisciplinario de programas de ingeniería multidisciplinares. Scientia et Technica, 18(1), 86-94.

Chetty, J. (2015). Lego Mindstorms: Merely a toy or a powerful pedagogical tool for learning computer programming?. In Proceedings de la 38 Australasian Computer Science Conference ACSC'15, 111-118. Recuperado de https://pdfs.semanticscholar. org/eo4f/23c697c48013ae2bd1c4c485ad015163fc34.pdf

Cooke, J. (2012). Everything you want to know about agile. Ely, Cambridgeshire, UK: IT Governance Publishing.

Corchuelo, M. (2015). Propuesta de lineamientos para el desarrollo de ambientes de aprendizaje en robótica a través del estudio de experiencias (Tesis de Maestría). Universidad de la Sabana, Colombia. Recuperado de http://hdl.handle. net/10818/20274 
Correll, N., Wailes, C., \& Slaby, S. (2014). A One-Hour Curriculum to Engage Middle School Students in Robotics and Computer Science, Using Cubelets. En: Ani Hsieh, M., \& Chirikjian, G. (eds), Distributed Autonomous Robotic Systems Springer Tracts in Advanced Robotics, vol 104 (pp. 165-176). doi: 10.1007/978-3-64255146-8_12

De Silva, C., \& Behbahani, S. (2013). A design paradigm for mechatronic systems. Mechatronics, 23(8), 960-966. doi: 10.1016/j.mechatronics.2012.08.004

Dode, P., Greig, M., Zolfaghari, S., \& Neumann, W. (2016). Integrating human factors into discrete event simulation: a proactive approach to simultaneously design for system performance and employees' well being. International Journal of Production Research, 54(10), 3105-3117. doi: 10.1080/00207543.2016.1166287

Dym, C., Agogino, A., Eris, O., Frey, D., \& Leifer, L. (2005). Engineering Design Thinking, Teaching and Learning. Journal of Engineering Education, 94(1), 103-120. doi: 10.1002/j.2168-9830.2005.tboo832.x

Edin Grimheden, M. (2013). Can agile methods enhance mechatronics design education? Mechatronics, 23(8), 967-973. doi: 10.1016/j.mechatronics.2013.01.003

García, Y., \& Reyes, D. (2012). Robótica educativa y su potencial mediador en el desarrollo de las competencias asociadas a la alfabetización científica. Revista Educación y Tecnología, 2, 42-55.

Gausemeier, J., Dorociak, P., Pook, S., Nyßen, A., \& Terfloth, A. (2010). Computer-aided cross-domain modeling of mechatronic systems. In Proceedings of Design 2010, the 11th International Design Conference, Dubrovnik, Croatia, 723-732. Recuperado de https://www.designsociety.org/download-publication/29417/computer-aided_ cross domain_modeling_of_mechatronic_systems

Ghitis, T., \& Alba, J. A. (2014). Los robots llegan a las aulas. Infancias imágenes, 13(1), $143-147$.

Gómez-González, C., Lecuona-López, M., \& Hernández-Monsalve, M. (2016). Análisis de bibliografía sobre políticas de diseño. Iconofacto, 12(19), 268-293. doi: 10.18566/ iconofact.v12.n19.a12

Guzey, S., Moore, T., \& Harwell, M. (2016). Building Up STEM: An Analysis of TeacherDeveloped Engineering Design-Based STEM Integration Curricular Materials. Journal of Pre-College Engineering Education Research (J-PEER), 6(1). doi: 10.7771/2157-9288.1129

Hassan, A., Siadat, A., Dantan, J., \& Martin, P. (2010). Conceptual process planning - an improvement approach using QFD, FMEA, and ABC methods. Robotics and Computer-Integrated Manufacturing, 26(4), 392-401. doi: 10.1016/j. rcim.2009.12.002

Isermann, R. (2008). Mechatronic systems - Innovative products with embedded control. Control Engineering Practice, 16(1), 14-29. doi: 10.1016/j.conengprac.2007.03.010 
Kuhn, W. (2006). Digital Factory - Simulation Enhancing the Product and Production Engineering Process. In Proceedings of the 2006 Winter Simulation Conference, IEEE, 1899-1906. doi: 10.1109/wsc.2006.322972

Kurdve, M., Zackrisson, M., Wiktorsson, M., \& Harlin, U. (2014). Lean and green integration into production system models - experiences from Swedish industry. Journal of Cleaner Production, 85, 180-190. doi: 10.1016/j.jclepro.2014.04.013

Macas, E. (2017). Definición y Estado del Arte de la Ingeniería Concurrente La Manufactura Integrada por Computador y la Mecatrónica. INNOVA Research Journal, 2(10), 44-6o. doi: 10.33890/innova.v2.n10.2017.286

Ministerio de Educación Nacional de Colombia (2008). Ser competente en tecnología: iUna necesidad para el desarrollo! Serie Guías No. 30: Orientaciones generales para la educación en tecnología. Recuperado de: https://www.mineducacion.gov. co/1621/articles-160915_archivo_pdf.pdf

Mizell, S., \& Brown, S. (2017). The Current Status of STEM Education Research 20132015. Journal of STEM Education, 17(4), 52-56. Recuperado de https://www. jstem.org/jstem/index.php/JSTEM/article/view/2169/1815

Monsalves, S. (2019). Estudio sobre la utilidad de la robótica educativa desde la perspectiva del docente. Revista de Pedagogía, 32(90),81-117.

Moreno, I., Muñoz, L., Serracín, J., Quintero, J., Patiño, K., \& Quiel, J. (2012). La robótica educativa, una herramienta para la enseñanza-aprendizaje de las ciencias y las tecnologías. Teoría de la Educación. Educación y Cultura en la Sociedad de la Información, 13(2), 74-90.

Odorico, A. (2005). La robótica desde una perspectiva pedagógica. Revista de Informática Educativa y Medios Audiovisuales, 2(5), 33-48.

Organización de los Estados Americanos (2016). La indagación como estrategia para la educación STEAM. Red Regional sobre Educación en Ciencia, Tecnología, Ingeniería, Artes y Matemáticas - EducaSTEAM. Recuperado de http://recursos. portaleducoas.org/sites/default/files/Final\%20OEA\%2oIndagacio\%CC\%81n.pdf

Pessôa, M., \& Trabasso, L. (2016). The Lean Product Design and Development Journey, 19-41. Cham, Switzerland: Springer International Publishing. doi: 10.1007/9783-319-46792-4_2

Pinto, M., Barrera, N., \& Pérez, W. (2010). Uso de la robótica educativa como herramienta en los procesos de enseñanza. Ingeniería Investigación Y Desarrollo, 10(1), 15-23.

Punz, S., Hehenberger, P., Follmer, M., \& Zeman, K. (2011). Customer-oriented hierarchical concept development - application of the HoC approach in mechatronic design. In 2011 IEEE International Conference on Mechatronics. doi: 10.1109/ icmech.2011.5971285 
Rivadeneira, P. (2019). La robótica como una herramienta para facilitar el aprendizaje y desarrollo de las competencias STEM en los integrantes del equipo de robótica Pólux de la Institución Educativa Juan Nepomuceno Cadavid (Tesis de Maestría). Universidad EAFIT, Medellín, Colombia. Recuperado de: http://hdl.handle. net/10784/12087

Sáez-Carreras, J., Bas-Peña, E., \& Bedmar-Moreno, M. (2007). Pedagogía social y educación social: historia, profesión y competencias. Madrid: Pearson Educación.

Salas-Lòpez, G., Sandoval-González, O., Herrera-Aguilar, I., Martínez-Sibaja, A., Portillo-Rodríguez, O., \& Vilchis-González, A. (2012). Design and development of a planar robot for upper extremities rehabilitation with visuo-vibrotactile feedback. Procedia Technology, 3, 147-156. doi: 10.1016/j.protcy.2012.03.016

Sánchez, M. (2003). Implementación de estrategias de robótica pedagógica en las instituciones educativas. In Blog Juan Carlos Sánchez Guerrero, Eduteka ICESI. Recuperado de: http://www.oocities.org/es/juancarlossanchezguerrero/ger/ii/ local/RoboticaPropuesta.pdf

Servicio Nacional de Aprendizaje (2015). Book Metodología de desarrollo de proyectos de base tecnológica. Bogotá: Sena Red Tecnoparque.

Silva-Ortigoza, R., Márquez-Sánchez, C., Carrizosa-Corral, F., Hernández-Guzmán, V., Garcia-Sánchez, J., Taud, H., Marciano-Melchor, M., \& Álvarez-Cedillo, J.A. (2014). Obstacle Avoidance Task for a Wheeled Mobile Robot - A Matlab-Simulink-Based Didactic Application. MATLAB. Applications for the Practical Engineer, 79-102. doi: $10.5772 / 58392$

Steimer, C., Fischer, J., \& Aurich, J. (2017). Model-based Design Process for the Early Phases of Manufacturing System Planning using SysML. In Procedia CIRP, 60, 163-168. doi: 10.1016/j.procir.2017.01.036

Vallejo-Jiménez, M. (2018). Diseño e implementación de prototipo de robot Lego Mindstorms EV3 para apropiación del concepto de la luz. Revista Tecnoacademia Con-ciencia y técnica, 2, 12-16. Recuperado de: http://revistas.sena.edu.co/index. $\mathrm{php} /$ conciencia/article/viewFile/1953/2072

Vallejo-Jiménez, M., Martínez-Puerta, J., Bedoya-Agudelo, S., \& Salgado, N. (2018). SENA Tecnoacademia Risaralda and Caldas as a Collaborative Learning Scenario in Robotics. En: Cristóbal J. Nettle, Miguel A. Solis (eds), Revista del 4th Congress on Robotics and Neuroscience, 39-45. Recuperado de https://crone2018.pubpub. org/pub/hdk7rpow 